\title{
Gas Diffusion through the Fractal Landscape of the Lung: How Deep Does Oxygen Enter the Alveolar System?
}

\author{
Chen $\mathrm{Hou}^{1)}$, Stefan Gheorghiu ${ }^{2}$, Marc-Olivier Coppens ${ }^{2)}$, Virginia H. Huxley ${ }^{3)}$, and Peter Pfeifer ${ }^{1)}$ \\ 1) Department of Physics, University of Missouri, Columbia, MO 65211, USA \\ 2) Department of Chemical Engineering, Delft University of Technology, Julianalaan 136, 2628 BL \\ Delft, The Netherlands \\ 3) Department of Physiology, University of Missouri, Columbia, MO 65211, USA
}

\begin{abstract}
Summary. We investigate oxygen transport to and across alveolar membranes in the human lung, the last step in the chain of events that takes oxygen through the bronchial airways to the peripheral, acinar airways. This step occurs by diffusion. We carry out analytic and numerical computations of the oxygen current for fractal, space-filling models of the acinus, based on morphological data of the acinus and appropriate values for the transport constants, without adjustable parameters. The computations address the question whether incoming oxygen reaches the entire available membrane surface (reaction-limited, unscreened oxygen current), a large part of the surface (mixed reaction/diffusion-limited, partly screened current), or only the surface near the entrance of the acinus (diffusion-limited, completely screened current). The analytic treatment identifies the three cases as sharply delineated screening regimes and finds that the lung operates in the partial-screening regime, close to the transition to no screening, for respiration at rest; and in the no-screening regime for respiration at exercise. The resulting currents agree well with experimental values. We test the analytic treatment by comparing it with numerical results for two-dimensional acinus models and find very good agreement. The results provide quantitative support for the conclusion, obtained in other work, that the space-filling fractal architecture of the lung is optimal with respect to active membrane surface area and minimum power dissipation. At the level of the bronchial tree, we show that the space-filling architecture provides optimal slowing down of the airflow from convection in the bronchial airways to diffusion in the acinar airways.
\end{abstract}

\section{Introduction}

One of the great promises of fractals in nature [1] is that they offer a powerful platform to study structure-function relationships of complex systems in science and engineering $[2,3,4]$. On the structural side, fractal geometry provides the simplest possible model of a complex system: the fractal dimension specifies the degree of irregularity or complexity, the inner cutoff specifies the size of the elementary building blocks, and the outer cutoff specifies the overall system size. These specifications are the necessary minimum for any meaningful parametrization. Remarkably, they are also sufficient in many instances: the functional properties of the system - the answer to the question, how does the system function?-often do not depend, in leading order, on how the system is constructed, as long as the fractal dimension, the inner and outer cutoff, and the composition are the same [5]. Thus fractal systems can predict functional properties with a high degree of universality and a minimum number of parameters. A case study of such universality for a whole series of structure-function relations is described in Refs. $[6,7]$. A single surface in that class can be tailored to perform multiple functions, each meeting a separate, preset performance target. Such opportunities for multiply optimized design, by appropriate design of a surface's geometry, are of outstanding interest in engineering $[8,9,10]$. 


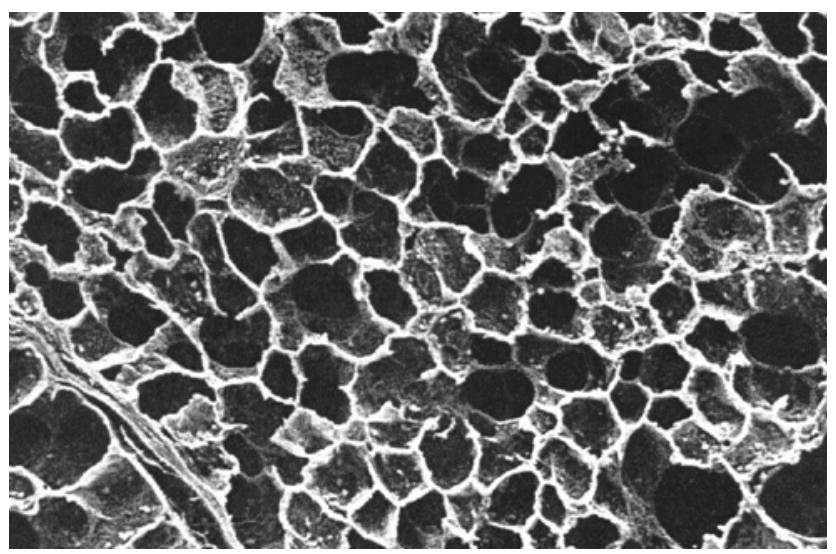

Figure 1. Space-filling system of alveoli in the human lung (from [11], with permission). The average diameter of an alveolus is $187 \mu \mathrm{m}$, and the number of alveoli is about 300 million. On scales larger than $187 \mu \mathrm{m}$, the walls between adjacent alveoli span a surface with fractal dimension 3 .

In this paper, we investigate structure-function relationships of the human lung and explore to what extent its fractal structure optimizes one or several functions of biological interest. Our focus is on oxygen transport, expressed as current across the cumulative membrane surface that separates alveoli and capillaries (air-blood barrier). The airways in the lung branch dichotomously over 23 generations, the first 14 of which are the bronchial airways, transporting air in and out by convection; the last 9 are the acinar airways, transporting air predominantly by diffusion $[12,13]$. Both the bronchial airways and the acinar airways have a well-defined, although slightly different, fractal structure. The bronchial airways form a tree whose "canopy" is space-filling [1, p. 157] and thus has fractal dimension 3. We show that this structure is optimal for efficient slowing down of the convective flow, preconditioning the air for diffusive transport in the acinar airways (Sect. 2). The acinar airways form a tree that is space-filling as a whole, the alveoli being the elementary building blocks and manifestly spanning a 3dimensional surface (Fig. 1). In Sects. 3-4, we compute the diffusion current of oxygen to and across the space-filling alveolar membrane system, based on an analytic treatment called rope-walk approximation (RWA), and complemented by finite-elementmethod (FEM) computations. We show that the current-and the answer to how deep the oxygen enters the alveolar system-depends critically on the competition between diffusion through the air space and transfer across alveolar walls. The competition unfolds into four rivaling length scales and power laws, controlled by the fractal dimension of the alveolar system, for the current as a function of the transport parameters. In Sects. 5-6, we discuss the results in terms of the multifaceted question whether the lung is an optimally designed gas exchanger. The results offer new perspectives for artificial lung technologies [14].

Previous studies of biological function in terms of the fractal structure of the bronchial airways include Mandelbrot [1], Weibel [12, 13, 15], West [16], Shlesinger and West [17], West et al. [18], and Mauroy et al. [19]. Studies in terms of the fractal structure of the acinar airways include Sapoval et al. [20, 21] and Felici et al. [22, 23]. 
To understand function of a biological system is a problem in reverse engineering. An approach to this problem has been formulated by Frauenfelder: "All present electronic devices work at room temperature, but the understanding of the solid-sate components required experiments over a wide range of temperatures. Biomolecules also work at ambient temperatures, but a full understanding of their dynamics and function also calls for experiments over a wide range temperatures [24]." Similarly, we investigate the function of the alveolar system by analyzing the oxygen current, in the RWA, over membrane permeabilities far beyond the physiological range. Indeed, the principal purpose of the RWA is: (a) to provide a practical formula that predicts the current at arbitrary values of the structural and transport parameters and thus serves as a laboratory to conduct 'in vitro experiments' over a wide range of variables; and (b) to provide a practical map, with clear-cut boundaries, of the regions in parameter space that correspond to currents controlled by diffusion through the air space, transfer across alveolar walls, or both. No such formula and map has been developed before.

\section{Slowing Down of Air in the Bronchial Tree}

In order for air to supply oxygen to the alveolar walls by diffusion, the flow velocity of air in the bronchial tree must be reduced to match the diffusion velocity in the acinar airways. As air moves through successive bifurcations of the bronchial tree into ducts of decreasing diameter, it slows down by virtue of the increasing cumulative crosssectional area of ducts. If the duct diameters before and after a bifurcation, $d, d_{1}$, and $d_{2}$ (Fig. 2), satisfy the relation

$$
d^{\Delta}=d_{1}^{\Delta}+d_{2}^{\Delta}
$$

where $\Delta$ is the tree diameter exponent [1], then the flow velocities before and after the bifurcation, $v, v_{1}$, and $v_{2}$, are given by

$$
v=\frac{d_{1}^{2} v_{1}+d_{2}^{2} v_{2}}{\left(d_{1}^{\Delta}+d_{2}^{\Delta}\right)^{2 / \Delta}},
$$

by mass conservation (equation of continuity at constant fluid density). For symmetric branching, $d_{1}=d_{2}=d_{\mathrm{b}}$ and $v_{1}=v_{2}=v_{\mathrm{b}}$, the diameter and velocity after the bifurcation, $d_{\mathrm{b}}$ and $v_{\mathrm{b}}$, are related to the diameter and velocity before the bifurcation by $d_{\mathrm{b}}=2^{-1 / \Delta} d$ and $v_{\mathrm{b}}=2^{-(\Delta-2) / \Delta} v$ from (1) and (2). In the bronchial tree, the bifurcations follow Murray's law, $d_{\mathrm{b}}=2^{-1 / 3} d$, for branching generation 0 (trachea) through 14 (transitional bronchioles) $[1,12,13,25,26]$. So the diameter exponent of the tree is $\Delta=3$, which makes the canopy (collection of all branch tips) space-filling and have fractal dimension 3 [1]. This constitutes the first part of the fractal landscape of the lung.

The expressions for $d_{\mathrm{b}}$ and $v_{\mathrm{b}}$ describe the diameter and velocity after one bifurcation. The diameter and velocity after $n$ bifurcations, $d_{\mathrm{b}}^{(n)}$ and $v_{\mathrm{b}}^{(n)}$, in terms of the initial data $d$ and $v$ (branching generation 0 ) are given by

$$
\begin{aligned}
& d_{\mathrm{b}}^{(n)}=2^{-n / \Delta} d, \\
& v_{\mathrm{b}}^{(n)}=2^{-n(\Delta-2) / \Delta} v .
\end{aligned}
$$




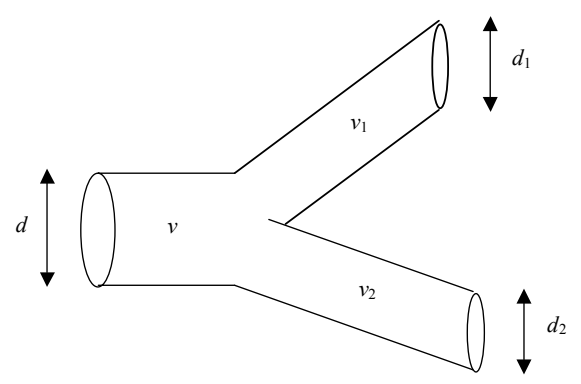

Figure 2. Bifurcation of ducts in the bronchial tree (schematic). Air flows with velocity $v$ through the parent duct of diameter $d$ and is split into flows of velocity $v_{1}$ and $v_{2}$ in the daughter branches with diameter $d_{1}$ and $d_{2}$.

If we ask that the velocity drop over $n$ bifurcations be maximum, so as to slow down the air to a prescribed value $v_{\mathrm{b}}^{(n)}$ over a minimum number of bifurcations, it follows from (4) that the maximum is attained when $1-(2 / \Delta)$ is maximum, i.e., when $\Delta=3$. A diameter exponent larger than three would lead to branch tips that overlap after a finite number of bifurcations [1], leaving no room for acinar airways at the periphery of the bronchial airways. For $\Delta=3$ and $n=14$, the velocity drop from (4) is $v_{\mathrm{b}}^{(n)} / v \approx 0.039$, and the diameter drop from (3) is $d_{\mathrm{b}}^{(n)} / d \approx 0.039$.

Why should an optimal design reduce the flow velocity in the airways, and seek to achieve the reduction through a minimum number of bifurcations? If the flow velocity $v_{\mathrm{b}}^{(n)}$ is larger than the diffusion velocity, $D / d_{\mathrm{b}}^{(n)}$ (duct diameter divided by the time it takes oxygen to diffuse across the duct, with $D$ the diffusion coefficient of oxygen in air), i.e., if the Peclet number $v_{\mathrm{b}}^{(n)} d_{\mathrm{b}}^{(n)} / D$ is larger than one, then significant oxygen concentration gradients exist and oxygen transport to the duct wall is suboptimal. Reduction of the flow velocity via a minimum number of bifurcations is optimal because the "hardware" required to build $n$ bifurcations, such as the surface area of the walls needed to form $1+2+\ldots+2^{n} \approx 2^{n+1}$ ducts, grows exponentially with $n$. An alternative view of the Peclet number compares the time to move a distance $d_{\mathrm{b}}^{(n)}$ by convection, $t_{\text {conv }}=d_{\mathrm{b}}^{(n)} / v_{\mathrm{b}}^{(n)}$, with the time to move the same distance by diffusion, $t_{\mathrm{diff}} \approx\left(d_{\mathrm{b}}^{(n)}\right)^{2} / D$. If the Peclet number is less than one, $t_{\text {diff }} / t_{\text {conv }}<1$, transport by diffusion is faster than transport by convection. So a Peclet number less than one after $n$ bifurcations is not only necessary for efficient transport, but also sufficient.

Thus, while Murray's work and that of others has found the space-filling design of the bronchial airways, with $\Delta=3$, to be optimal with respect to energy costs (minimum energy dissipation) $[1,12,13,19,25,26]$, here we see that $\Delta=3$ also generates an optimal chemical reactor-gradient-free, well-stirred, at minimum hardware costs - as the starting point for diffusion of oxygen through the acinar airways.

The condition that the Peclet number should be less than one for a gradient-free reservoir translates into

$$
\frac{v_{\mathrm{b}}^{(n)} d_{\mathrm{b}}^{(n)}}{D}=2^{-n(\Delta-1) / \Delta} \frac{v d}{D}<1
$$

by Eqs. (3) and (4). This shows that the Peclet number decreases most rapidly over $n$ 
bifurcations if $1-(1 / \Delta)$ is maximum, i.e., if $\Delta=3$, in agreement with the result from the maximum velocity drop over $n$ bifurcations. The volume flow rate of air (total ventilation) in the human lung is $1.3 \times 10^{2} \mathrm{~cm}^{3} / \mathrm{s}[12, \mathrm{p} .283]$; the diameter of the trachea is $d=1.8 \mathrm{~cm} \mathrm{[12,} \mathrm{p.} \mathrm{278],} \mathrm{resulting} \mathrm{in} \mathrm{a} \mathrm{flow} \mathrm{velocity} \mathrm{in} \mathrm{the} \mathrm{trachea} \mathrm{of} v=51 \mathrm{~cm} / \mathrm{s}$; and the diffusion coefficient of oxygen in air is $D=0.24 \mathrm{~cm}^{2} / \mathrm{s}$ (Sect. 4). From these data and $\Delta=3$, Eq. (5) predicts $n>13$ (rounded to the nearest integer) for the number of bifurcations necessary for the bronchial airways to generate a gradient-free oxygen reservoir. This is remarkably close to the observed number of bifurcations, $n=14$, for the observed exponent $\Delta=3$. It is a strong example of symmorphosis [13], mediated by fractal geometry: the exponent $\Delta=3$ and the 14 bifurcations are perfectly matched to slow down the air - under the given ventilation rate, diameter of the trachea, oxygen diffusivity, and minimal manufacturing (hardware) and maintenance (energy) costs - to the point where it forms a well equilibrated reservoir for oxygen diffusion.

Estimates of the Peclet number and the convection-diffusion transition, $n=18$ for respiration at rest and $n=21$ for respiration at exercise, based on data that includes the structure of the acinar airways, can be found in Ref. [21].

\section{Designing an Efficient Oxygen Receptor}

With a well-equilibrated oxygen reservoir at hand, how should the subsequent ducts and membrane system (acini, alveoli) be structured for efficient oxygen diffusion to and across the membrane? Should the ducts be long and thin, as suggested by Adam and Delbrück's celebrated result that diffusing molecules find a given receptor most rapidly if the diffusion space is one-dimensional [27]? Should the alveoli span a large cumulative perimeter, as suggested by Kac's result that a three-dimensional diffusion space is depleted most rapidly by a large number of spherical absorbers when their cumulative perimeter diverges $[28,29]$ ? Or should the alveoli simply span a large cumulative surface area?

There exists a rich body of work on structure-function relations for diffusion of molecules to biological receptors and capture thereat, with unexpectedly varied conclusions (Table 1). Some of these relations are unexpected because they predict capture rates proportional to the receptor perimeter instead of area (Entries 3-5); others, comparing capture in diffusion spaces of different dimensionality, depend on whether the diameter or volume of the space is kept constant in the comparison (Entries 1,2). So there is no simple one-size-fits-all design of an optimal receptor. The reason, illustrated in the table, is that capture rates depend sensitively on what structural parameters (nature of the source, distance between source and receptor, shape of the receptor) are allowed to vary in the optimization. The sensitivity arises because capture depends on the probability $p$ that the molecule's trajectory hits the receptor surface. For a spherical receptor of radius $R$ in 3 dimensions, $p$ scales as $p \propto R^{2-D_{\mathrm{f}, \text { int }}}$, where $D_{\mathrm{f} \text {,int }}$ is the fractal dimension of the intersection of the trajectory and the surface. If the molecule arrives from a distant source, its trajectory is a Brownian path and has fractal dimension 2, which gives $D_{\mathrm{f} \text {,int }}=1$ and $p \propto R$. If the molecule arrives from a close source, such as a gradient-free reservoir in contact with the surface, the trajectory is effectively 1-dimensional, which gives $D_{\mathrm{f} \text {,int }}=0$ and $p \propto R^{2}$. The two cases correspond to the capture rates proportional to receptor perimeter and surface area, respectively, in Table 1. 
Table 1. Models for diffusion to biological receptors. The diffusion space is a large $d$-dimensional sphere. The permeability, $W$, is the number of molecules crossing the membrane per unit time, surface area, and concentration difference between the two sides of the membrane.

\begin{tabular}{|l|l|l|l|l|}
\hline & $\begin{array}{l}\text { Diffusion } \\
\text { space }\end{array}$ & Source & Receptor & Optimal design \\
\hline $\begin{array}{l}\text { 1. Adam-Delbrück } \\
\text { model [27, 8] }\end{array}$ & $\begin{array}{l}\text { Fixed diame- } \\
\text { ter; } d=1,2,3\end{array}$ & Diffusion space & $\begin{array}{l}\text { Small sphere; } \\
W=\infty\end{array}$ & $\begin{array}{l}\text { Mean diffusion time to reach } \\
\text { receptor is minimum if } d=1\end{array}$ \\
\hline $\begin{array}{l}\text { 2. Inverse Adam- } \\
\text { Delbrück model [8] }\end{array}$ & $\begin{array}{l}\text { Fixed volume; } \\
d=1,2,3\end{array}$ & Diffusion space & $\begin{array}{l}\text { Small sphere; } \\
W=\infty\end{array}$ & $\begin{array}{l}\text { Mean diffusion time to reach } \\
\text { receptor is minimum if } d=3\end{array}$ \\
\hline $\begin{array}{l}\text { 3. Kac' theorem [28, } \\
\text { 29] }\end{array}$ & $\begin{array}{l}\text { Fixed diame- } \\
\text { ter; } d=3\end{array}$ & Diffusion space & $\begin{array}{l}\text { Many small } \\
\text { spheres; } W=\infty\end{array}$ & $\begin{array}{l}\text { Diffusion space is depleted } \\
\text { most rapidly if cumulative } \\
\text { perimeter of spheres diverges }\end{array}$ \\
\hline $\begin{array}{l}\text { 4. Berg-Purcell } \\
\text { chemoreceptor [30] }\end{array}$ & $\begin{array}{l}\text { Fixed diame- } \\
\text { ter; } d=3\end{array}$ & $\begin{array}{l}\text { Boundary of } \\
\text { diffusion space }\end{array}$ & $\begin{array}{l}\text { Many small } \\
\text { disks on host } \\
\text { sphere; } W=\infty\end{array}$ & $\begin{array}{l}\text { Capture probability is } \approx \text { if } \\
\text { cumulative perimeter of disks } \\
\text { is }>>\text { perimeter of host sphere }\end{array}$ \\
\hline $\begin{array}{l}\text { 5. Diffusion-limited } \\
\text { receptor; Eq. (6a) }\end{array}$ & $\begin{array}{l}\text { Fixed diame- } \\
\text { ter; } d=3\end{array}$ & $\begin{array}{l}\text { Boundary of } \\
\text { diffusion space }\end{array}$ & $\begin{array}{l}\text { Small sphere; } \\
W=\infty\end{array}$ & $\begin{array}{l}\text { Capture rate is proportional to } \\
\text { perimeter } \text { of sphere }\end{array}$ \\
\hline $\begin{array}{l}\text { 6. Reaction-limited } \\
\text { receptor; Eq. (6b) }\end{array}$ & $\begin{array}{l}\text { Fixed diame- } \\
\text { ter; } d=3\end{array}$ & $\begin{array}{l}\text { Boundary of } \\
\text { diffusion space }\end{array}$ & $\begin{array}{l}\text { Small sphere; } \\
W \rightarrow 0\end{array}$ & $\begin{array}{l}\text { Capture rate is proportional to } \\
\text { surface area } \text { of sphere }\end{array}$ \\
\hline $\begin{array}{l}\text { 7. Makarov's } \\
\text { theorem [31, 32, 33] }\end{array}$ & $\begin{array}{l}\text { Fixed diame- } \\
\text { ter; } d=2\end{array}$ & $\begin{array}{l}\text { Boundary of } \\
\text { diffusion space }\end{array}$ & $\begin{array}{l}\text { Large irregular } \\
\text { surface; } W=\infty\end{array}$ & $\begin{array}{l}\text { Capture rate is proportional to } \\
\text { diameter } \text { of receptor }\end{array}$ \\
\hline
\end{tabular}

We treat oxygen transport in the acinar airways as a stationary diffusion-reaction process. The oxygen concentration $c(\mathbf{x})$ obeys Laplace's equation, $\nabla^{2} c(\mathbf{x})=0$, at position $\mathbf{x}$ in the diffusion space; $c(\mathbf{x})=c_{0}$ for $\mathbf{x}$ at the entrance to the diffusion space, with $c_{0}$ the concentration in the gradient-free reservoir; and $D \nabla c(\mathbf{x}) \cdot \mathbf{n}(\mathbf{x})=W\left(c(\mathbf{x})-c_{1}\right)$ for $\mathbf{x}$ at the alveolar membrane surface, where $\mathbf{n}(\mathbf{x})$ is the surface normal pointing into the diffusion space, $c_{1}$ is the oxygen concentration in the blood, and $D$ and $W$ have been introduced earlier. The boundary condition at the membrane equates the bulk diffusion flux to the transmembrane flux. The oxygen current, $I$, across the alveolar surface is obtained from $I=\int_{\text {surface }} W\left(c(\mathbf{x})-c_{1}\right) d S$ where $S$ is surface area.

If the diffusion space and membrane are spherical and concentric with radius $R+a$ and $R$, respectively, and the source with concentration $c_{0}$ (gradient-free reservoir) is at $R+a$, the diffusion-reaction problem has the elementary solution

$$
I=4 \pi R D\left(c_{0}-c_{1}\right)\left(\frac{D}{W R}+\frac{a}{a+R}\right)^{-1} \stackrel{a \rightarrow \infty}{\sim} \begin{cases}4 \pi R D\left(c_{0}-c_{1}\right) & \text { for } W \rightarrow \infty \\ 4 \pi R^{2} W\left(c_{0}-c_{1}\right) & \text { for } W \rightarrow 0 .\end{cases}
$$

This structure-function relation, pedagogical and nonfractal as it is, illustrates the main features of oxygen diffusion in the acinus, if we equate $R$ to the size of a given alveolar region, and $a$ to the path length from the entrance of the diffusion space to the region in question. The exploration length, $D / W$, is a measure of the size of the surface region an incoming molecule explores before it crosses the membrane. Regions within path length $D / W$ from the entrance act like a reaction-limited receptor [Eq. (6b), valid whenever $D / W>\max \{a, R\}$, contributing a current proportional to $R^{2} W$, i.e., to surface area and permeability. Such a region is unscreened because it is accessible to incoming oxygen without significant concentration drop, $c(\mathbf{x}) \approx c_{0}$. Regions more distant from the entrance act like a diffusion-limited receptor [Eq. (6a), valid whenever 
$D / W<<\min \{a, R\}]$, contributing a current that is negligible by virtue of $R D<<R^{2} W$. Such a region is screened because it is reached only by few molecules, resulting in a large concentration drop, $c(\mathbf{x})<<c_{0}$.

If we vary the permeability from large to small, the exploration length changes from small to large, taking the surface from complete to partial to no screening, according to whether all, some, or no regions are screened. Thus an efficient oxygen receptor, "wasting" no alveolar surface area, should be in the no-screening regime.

\section{Oxygen Current Across the Space-Filling System of Alveoli}

We model the membrane as a self-similar surface with fractal dimension $D_{\mathrm{f}}$, surface area $S$, and cubic elementary units of side length $\ell$. The source is taken as a surface segment, with area $S_{\mathrm{s}}$, of the smallest cube circumscribing the membrane. The cubic shape is merely for visualization and mathematical simplicity.

In previous work $[32,33]$, we have computed the current for the case where the source is the entire circumscribing cube, in the rope-walk approximation (RWA). In the RWA, one takes a surface profile in $d=2$, considers the surface sites that contribute to the current if the exploration length is zero (exposed sites; active zone at $W=\infty$ ), walks along the profile from any such site and "rolls out a rope" of length $D / W$ (active zone at $W<\infty$ ), and determines the length of the profile so covered by ropes. This length, when multiplied by $W$ and the concentration difference $c_{0}-c_{1}$, gives the current. The result can be lifted to $d=3$, giving

$$
I=W\left(c_{0}-c_{1}\right) \begin{cases}S_{\mathrm{s}} & \text { if } D / W \leq \ell, \\ S_{\mathrm{s}}[D /(W \ell)]^{\left(D_{\mathrm{f}}-2\right) /\left(D_{\mathrm{f}}-1\right)} & \text { if } \ell \leq D / W \leq \ell\left(S / S_{\mathrm{s}}\right)^{\left(D_{\mathrm{f}}-1\right) /\left(D_{\mathrm{f}}-2\right)}, \\ S & \text { if } \ell\left(S / S_{\mathrm{s}}\right)^{\left(D_{\mathrm{f}}-1\right) /\left(D_{\mathrm{f}}-2\right)} \leq D / W,\end{cases}
$$

for $2 \leq D_{\mathrm{f}} \leq 3$. The areas $S$ and $S_{\mathrm{s}}$ scale with $L$, the side length of the circumscribing cube, as $(L / \ell)^{D_{\mathrm{f}}} \ell^{2}$ and $L^{2}$, showing that (7) is well-behaved in the limit $D_{\mathrm{f}}=2$. The power laws for the current are the asymptotic expressions far from the crossover points, extended all the way to where the expressions intersect. This approximates the gradual crossover from one power law to another by an abrupt crossover.

The ropes of length $D / W$ stake out the surface regions a molecule explores before it crosses the membrane; the bracket in (7) is the correspondingly active, effective surface area. The ropes decompose the surface into regions accessible to the molecule, and regions inaccessible. The decomposition creates a map with sharp boundaries, which are determined by how the rope length compares with other lengths: In (7a), the rope length is smaller than an elementary building block of the surface, and only regions facing the source, with area $S_{\mathrm{s}}$, contribute to the current. This is the case of complete screening. In ( $7 b)$, the rope is long enough that incoming molecules enter the hierarchy of small and large fjords of the fractal surface, but not long enough for the molecules to visit the entire surface. This is the partial screening regime. In (7c), the rope length exceeds the perimeter of the surface profile, and the molecules visit the entire surface before they cross the membrane. This is the case of no screening. As we progress through $(7 \mathrm{a}-\mathrm{c})$ for decreasing $W$, the effective surface area stays constant $[(7 \mathrm{a}, \mathrm{c})]$ or increases $[(7 b)]$; but the current decreases, linearly $[(7 a, c)]$ or nonlinearly $[(7 b)]$. 


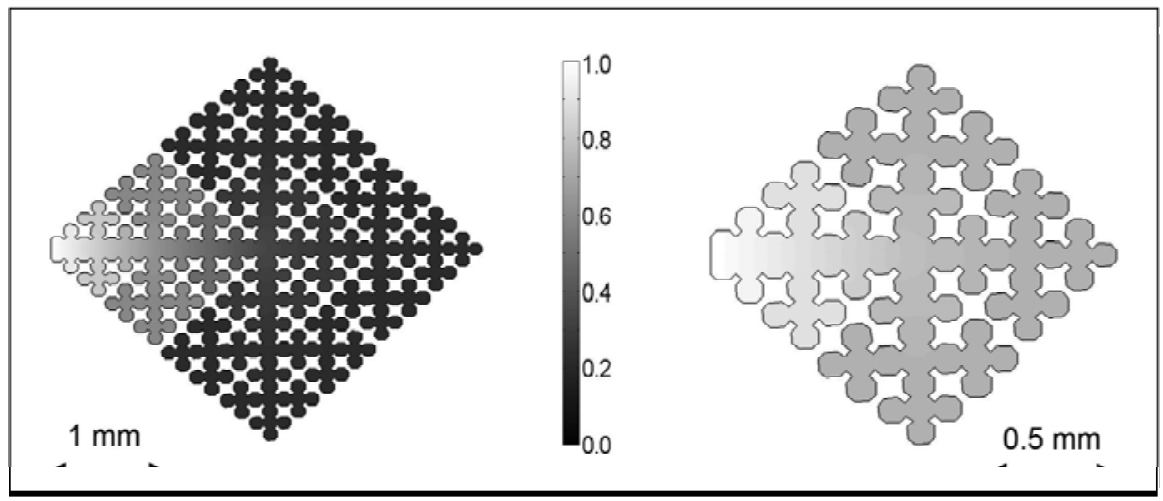

Figure 3. Oxygen concentration, $\left(c(\mathbf{x})-c_{1}\right) /\left(c_{0}-c_{1}\right)$, from FEM calculations, in two models of the acinar airways. Sierpinski's space-filling curve with 4 (left) and 3 (right) branching generations models planar cuts of the diffusion space for respiration at rest and exercise, respectively. The model and transport parameters are described in Table 2.

The increase in effective surface area does not offset the decrease in current due to the decrease in permeability.

The RWA has been tested extensively and agrees well with finite-elementmethod (FEM) solutions of Laplace's equation subject to the stated boundary conditions $[34,35]$. Because FEM solutions in $d=3$ are computationally intensive, most tests have been performed in $d=2$. No studies have been performed, however, for situations where the source is small compared to the circumscribing cube.

We present such a study in Figs. 3 and 4, for oxygen currents from FEM and RWA computations for two planar models of the space-filling alveolar system. The models represent the membrane surface by Sierpinski's space-filling curve [36] (Fig. 3) and treat the case of respiration at rest and at exercise, for which the diffusion space is $1 / 8$ and $1 / 128$ of an acinus, respectively [21].

The RWA, for $d=2,1 \leq D_{\mathrm{f}} \leq 2$, surface perimeter $S^{\prime}$, source perimeter $S_{\mathrm{s}}^{\prime}$, and small source - corresponding to a planar model, with arbitrary fractal dimension and narrow entrance, of the alveolar system — gives the current

$$
I=W\left(c_{0}-c_{1}\right) \begin{cases}S_{\mathrm{s}}^{\prime} & \text { if } D / W \leq \ell, \\ S_{\mathrm{s}}^{\prime}[D /(W \ell)]^{\left(D_{\mathrm{f}}-1\right) / D_{\mathrm{f}}} & \text { if } \ell \leq D / W \leq \ell\left(S_{\mathrm{s}}^{\prime} / \ell\right)^{D_{\mathrm{f}}} \\ D / W & \text { if } \ell\left(S_{\mathrm{s}}^{\prime} / \ell\right)^{D_{\mathrm{f}}} \leq D / W \leq S^{\prime} \\ S^{\prime} & \text { if } S^{\prime} \leq D / W .\end{cases}
$$

To appreciate the difference between (8) and (7), we first note that here $S^{\prime}$ and $S_{\mathrm{s}}^{\prime}$ are lengths instead of areas, and concentrations are per area instead of volume. Second, the source no longer surrounds the entire membrane, but only a small part of it. Because the source is small, we now have four independent length scales: the rope length $D / W$, inner cutoff $\ell$, outer cutoff $L$, and source perimeter $S_{\mathrm{s}}^{\prime}$. This gives rise to four screening regimes: complete screening, partial screening I, partial screening II, and no screening $[(8 \mathrm{a}-\mathrm{d})]$. The new regime, partial screening II, occurs when a single rope can cover a 

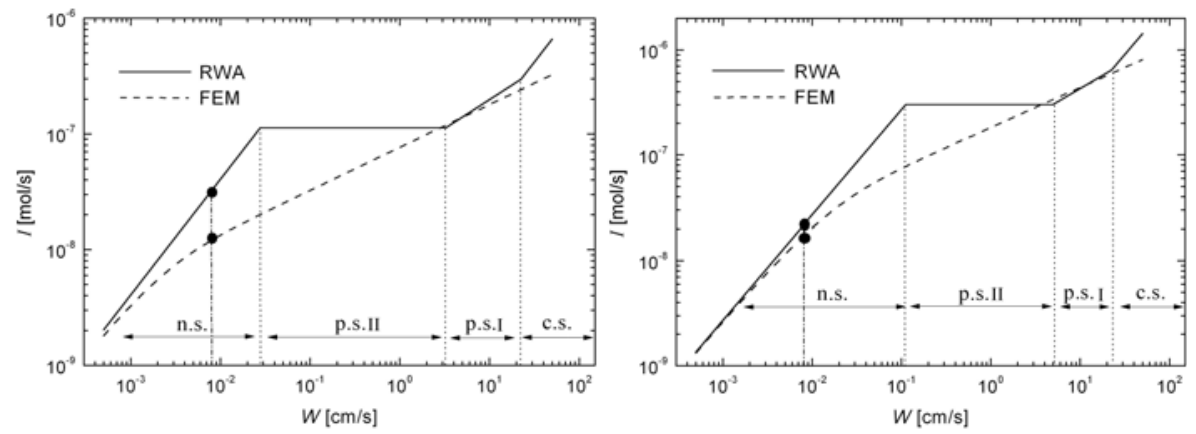

Figure 4. Oxygen currents from FEM and RWA computations, as a function of the permeability, for the models in Fig. 3. Left: respiration at rest. Right: respiration at exercise. The regimes of no screening (n.s.), partial screening (p.s.) I and II, and complete screening (c.s.) are explained in the text. The dots mark the currents at the physiological value of the permeability.

surface region larger than the source, $(D / W)^{1 / D_{\mathrm{f}}} \ell>S_{\mathrm{s}}^{\prime}$, but not the whole surface, $D / W<S^{\prime}$. In this case, the active zone consists of the few surface points facing the source (active zone at $W=\infty$ ), from each of which a rope of length $D / W$ is rolled out, and the active zone has length proportional to $D / W$, Eq. (8c). This creates the plateau, $I=$ const, at intermediate values of $W$ in Fig. 4. Specifically, the active zone in the Sierpinski subacinus at partial screening II consists of two ropes, starting at the upper and lower point of the entrance and tracing out the upper and lower periphery of the subacinus. Clearly, partial screening II and the associated plateau in the $I$ vs. $W$ curve exists only if the source is small. It is a characteristic of diffusion-reaction processes driven by a small source that has not been identified before.

Table 2. Structural and transport parameters for the models in Fig. 3, and resulting currents. The experimental values for $\ell$ and $L$ are $\ell=4 V_{\mathrm{a}} / S_{\mathrm{a}}$ and $L=\left(V_{\mathrm{a}} / 8\right)^{1 / 3},\left(V_{\mathrm{a}} / 128\right)^{1 / 3}$, with $V_{\mathrm{a}}$ and $S_{\mathrm{a}}$ the acinus volume and surface area [21]. In the models, $\ell$ is the experimental value; the number of branching generations, $m$ (unrelated to $n$ in Sect. 2), is chosen so that $L$ best matches the experimental value; and the ducts are tapered so that $S_{\mathrm{s}}^{\prime}$ matches the experimental side length of the subacinus entrance [37]. The values for $c_{0}-c_{1}$ are from partial pressure differences [12, $\mathrm{p}$. 361]. The values for $D$ and the physiological permeability $W_{\mathrm{p}}$, calculated from appropriate physical and biological data, are close to those in Ref. [21].

\begin{tabular}{|c|c|c|c|c|}
\hline & $\begin{array}{l}\text { At rest, } \\
\text { experimental }\end{array}$ & $\begin{array}{l}\text { At rest, } \\
\text { Sierpinski model }\end{array}$ & $\begin{array}{l}\text { At exercise, } \\
\text { experimental }\end{array}$ & $\begin{array}{l}\text { At exercise, } \\
\text { Sierpinski model }\end{array}$ \\
\hline Diffusion space & $1 / 8$ acinus & $m=4$ & $1 / 128$ acinus & $m=3$ \\
\hline Side length of alveolus, $\ell$ & \multicolumn{4}{|c|}{$0.0108 \mathrm{~cm}$} \\
\hline Side length of subacinus, $L$ & $0.286 \mathrm{~cm}$ & $0.244 \mathrm{~cm}$ & $0.114 \mathrm{~cm}$ & $0.122 \mathrm{~cm}$ \\
\hline Subacinus perimeter, $S^{\prime}$ & - & $8.69 \mathrm{~cm}$ & - & $2.17 \mathrm{~cm}$ \\
\hline Source perimeter, $S_{\mathrm{s}}^{\prime}$ & \multicolumn{2}{|c|}{$0.0283 \mathrm{~cm}$} & \multicolumn{2}{|c|}{$0.0219 \mathrm{~cm}$} \\
\hline Concentration, $c_{0}-c_{1}$ & $0.466 \mu \mathrm{mol} / \mathrm{cm}^{3}$ & $0.466 \mu \mathrm{mol} / \mathrm{cm}^{2}$ & $1.24 \mu \mathrm{mol} / \mathrm{cm}^{3}$ & $1.24 \mu \mathrm{mol} / \mathrm{cm}^{2}$ \\
\hline Diffusion coefficient, $D$ & \multicolumn{4}{|c|}{$0.243 \mathrm{~cm}^{2} / \mathrm{s}$} \\
\hline Permeability, $W_{\mathrm{p}}$ & \multicolumn{4}{|c|}{$0.00807 \mathrm{~cm} / \mathrm{s}$} \\
\hline FEM current at $W=W_{\mathrm{p}}$ & - & $0.0121 \mu \mathrm{mol} / \mathrm{s}$ & - & $0.0173 \mu \mathrm{mol} / \mathrm{s}$ \\
\hline RWA current at $W=W_{\mathrm{p}}$ & - & $0.0327 \mu \mathrm{mol} / \mathrm{s}$ & - & $0.0218 \mu \mathrm{mol} / \mathrm{s}$ \\
\hline
\end{tabular}




\section{$5 \quad$ Results}

We have computed the currents in Fig. 4 from the input data in Table 2. Both the FEM and RWA currents are absolute, without adjustable parameters. The currents agree within a factor of order one over 5 orders of magnitude of the permeability and nearly 3 orders of magnitude of the current. This is a remarkable agreement considering the fact that the FEM is exact, and the RWA is based solely on the evaluation of (8) with $D_{\mathrm{f}}=2$ and the listed values for $\ell, S^{\prime}$, and $S_{\mathrm{s}}^{\prime}$-without structural input, such as shapedependent prefactors, that would distinguish the Sierpinski model from any other spacefilling surface with the same $\ell, S^{\prime}$, and $S_{\mathrm{s}}^{\prime}$. It makes (8) a strong example for a fractal structure-function relation predicting a functional property, here the current, with a high degree of universality and a minimum number of parameters.

At the physiological permeability, $W=0.00807 \mathrm{~cm} / \mathrm{s}$, the ratio of RWA to FEM current is 2.7 and 1.3 at rest and exercise, respectively (Table 2), and both RWA currents are in the no-screening regime (Fig. 4). Equivalently, the FEM current is $37 \%$ and $79 \%$ of the maximum value, $I_{\max }=W\left(c_{0}-c_{1}\right) S^{\prime}$, at rest and exercise, respectively; and the RWA current is $100 \%$ of the maximum value in both cases. The ratio $\eta=I / I_{\max }$ represents the respiratory efficiency and is compared in Table 3 with results from other investigations. The table shows that FEM results from different models may differ by as much as a factor of 1.6 (Sierpinski vs. Hilbert model), which sets a lower bound for how closely the RWA can be expected to agree with exact results.

Thus in the framework of the RWA and the Sierpinski models, the oxygen enters the alveolar system completely, or $8.69 \mathrm{~cm}$ and $2.17 \mathrm{~cm}$ deep $\left(S^{\prime}\right)$ at rest and exercise. These depths are small fractions of the exploration length, $D / W=30.1 \mathrm{~cm}$, which implies that the oxygen visits even remote alveoli multiple times before it crosses the membrane. In contrast, at a permeability of, say, $0.200 \mathrm{~cm} / \mathrm{s}$, the RWA current would lie in the regime of partial screening II and the oxygen would enter the alveolar system to a depth of $D / W=1.22 \mathrm{~cm}$ in both models (Fig. 4). In this way, the FEM and RWA provide complementary information. The FEM states that oxygen enters the alveolar system to $37 \%$ and $79 \%$ at $W=0.00807 \mathrm{~cm} / \mathrm{s}$, and $5 \%$ and $20 \%$ at $W=0.200 \mathrm{~cm} / \mathrm{s}$. The RWA translates these fractions into path lengths that describe how deep the oxygen enters the subacinus, compares the path lengths to other lengths in the problem, and elaborates the effect on the current. At partial screening II, the path length is larger than the length along a surface segment equal in size to the entrance and gives a constant current; at partial screening I, the path length is shorter and gives a rising current; at complete screening the path length is less than the size of a single alveolus and gives a current limited to the immediate vicinity of the entrance.

The decomposition of the current into disjoint screening regimes provides a map to explore how alternative structures would perform at the given permeability, or how the given structure would perform at different permeabilities. E.g., in the model for the $1 / 8$ acinus, $W \sim 0.03 \mathrm{~cm} / \mathrm{s}$ marks the onset of "waste of acinar area", and $W \sim 2 \mathrm{~cm} / \mathrm{s}$ marks the onset of "waste of alveolar area". The current in this regime is at or near the value for partial screening II, $I=D\left(c_{0}-c_{1}\right)$, which is completely independent of the structure of the subacinus. This suggests that the subacinus is designed to operate at low permeability and high surface perimeter, rather than high permeability and low perimeter: for a perimeter less than $8.69 \mathrm{~cm}$, the line of no screening in Fig. 4 would be shifted to the right while the line of partial screening II stays put, allowing the target 
Table 3. Respiratory efficiency, $\eta$, for various models of the acinar airways and $D, W$ values identical or close to those in Table 2. The currents in the last two entries are discussed in the text.

\begin{tabular}{|l|l|l|l|}
\hline & & $\begin{array}{l}\text { At rest } \\
(1 / 8 \text { acinus })\end{array}$ & $\begin{array}{l}\text { At exercise } \\
(1 / 128 \text { acinus })\end{array}$ \\
\hline$d=2$ & $\eta$, Hilbert model; FEM [21] & $23 \%$ & $64 \%$ \\
& $\eta$, Kitaoka model; FEM [22] & $\sim 25 \%$ & $\sim 65 \%$ \\
& $\eta$, Sierpinski model; FEM & $37 \%$ & $79 \%$ \\
& $\eta$, Sierpinski model; RWA & $100 \%$ (n.s.) & $100 \%$ (n.s.) \\
\hline$d=3$ & $\eta$, Kitaoka model; random walk simulation $[23]$ & $33 \%$ & $\sim 100 \%$ \\
& $\eta$, morphological data for $S, S_{\mathrm{s}}, \ell ;$ RWA & $10 \%(\mathrm{p} . \mathrm{s.}$ II) & $100 \%$ (n.s.) \\
& $I_{\text {lung }}$, morphological data for $S, S_{\mathrm{s}}, \ell ;$ RWA & $0.743 \mathrm{mmol} / \mathrm{s}$ & $20.1 \mathrm{mmol} / \mathrm{s}$ \\
& $I_{\text {lung, } \text {, experimental [12] }}$ & $0.208 \mathrm{mmol} / \mathrm{s}$ & $1.80 \mathrm{mmol} / \mathrm{s}$ \\
\hline
\end{tabular}

current of $0.0327 \mu \mathrm{mol} / \mathrm{s}$ to be recovered at a higher permeability, all other conditions being the same. The fact that nature has not chosen this option suggests that it is more important to keep the permeability low than to save "hardware".

The outcome that the RWA current in the $1 / 8$ acinus is nearly constant, at the value $D\left(c_{0}-c_{1}\right)$, over almost 3 orders of magnitude of the permeability, explains why the FEM current varies by barely more than 1 order of magnitude. It highlights the nonlinear $I-W$ characteristic of the space-filling membrane. The value $D\left(c_{0}-c_{1}\right)$ reveals that the constant current along the plateau in Fig. 4 is diffusion-limited; it is the planar version of the diffusion-limited current (6a). The emergence of a diffusion-limited current flanked by a reaction-limited current on the left, $I=\left(c_{0}-c_{1}\right) S^{\prime} W$, and a mixed reaction/diffusion-limited current on the right, $I=\left(c_{0}-c_{1}\right) S_{\mathrm{s}}^{\prime}(D W)^{1 / 2}$, is unusual. The usual order, as $W$ increases, is: reaction-limited, reaction/diffusion-limited, diffusionlimited [Eq. (6)]. The current is diffusion-limited whenever the source is far from the receptor: in (6a) the source is distant by virtue of $D / W<<\min \{a, R\}$; in (8c) the source is distant because the incoming oxygen explores surface regions large compared to the size of the source, but not the entire surface. This shows that the diffusion-limited current in Fig. 4 is a consequence of the narrow entrance of the subacinus.

As a result of the values for $\ell, S^{\prime}, S_{\mathrm{s}}^{\prime}$, the power laws for partial screening I in the models extend over permeability intervals less than a decade, short of meeting the rule of thumb that a well-defined fractal power law should extend over length scales of a decade or more. Also, the ratio $L / \ell$ of 11 and 22 for the $1 / 128$ and $1 / 8$ acinus model, respectively, is on the low side to support several consecutive fractal power laws. Therefore, the RWA currents for partial screening I and complete screening in Fig. 4 should be regarded as idealizations that may be subject to corrections. But they agree extraordinarily well with the FEM currents, as the figure shows.

How do these results translate to $d=3$ ? We have extended the RWA for the case where the source is small to $d=3$. The remarkable performance of the RWA for the Sierpinski models suggests that the extension should be a good predictor of the oxygen current across the space-filling membrane in three dimensions. The results, calculated from the morphological data for $S, S_{\mathrm{s}}, \ell$ quoted in Table 2, validate this expectation (Table 3): the respiratory efficiency agrees within a factor of order one with recent simulations of oxygen transport in acinar models in $d=3$ [23]. In fact, the comparison with the efficiencies from simulations suggests that the accuracy of the RWA gets better as we go from two to three dimensions. A notable surprise is that the RWA 
current for the $1 / 8$ acinus switches from no screening to partial screening II as we go from two to three dimensions. The origin and significance of this result will be discussed elsewhere. Operation of the lung at partial screening II implies that the oxygen enters deep into the alveolar system-exploring surface regions much larger than the entrance to the subacinus, but not the entire surface - at rest.

Finally, we have used the RWA to calculate the oxygen currents for the whole lung and compare them with experimental values (Table 3 ). The calculated and experimental values agree within a factor of 4 and 11 at rest and exercise, respectively. While this is less than impressive on an absolute scale, it is impressive from the perspective of fractal scaling, based on only three structural parameters $\left(S, S_{\mathrm{s}}, \ell\right)$, three transport parameters $\left(D, W, c_{0}-c_{1}\right)$, and prefactors in which shape-dependent constants of order one have been set equal to one. Uncertainties in any of these can easily add up to an uncertainty of one order of magnitude in the current. For instance, our value $W=0.00807 \mathrm{~cm} / \mathrm{s}$ is the permeability of the membrane, for oxygen crossing the tissue barrier and blood plasma; it does not include the barrier for binding of oxygen in the red blood cells. No permeability values including the barrier for binding are known. So our permeability and currents are necessarily larger than the actual permeability and currents, and further work is needed to get more accurate estimates.

\section{Discussion}

We have shown how the space-filling structure of the bronchial airways sets up an optimal, well equilibrated reservoir for oxygen diffusion through the acinar airways, and how the space-filling structure of the acinar airways provides a network for efficient oxygen diffusion across the alveolar membranes, with almost all membrane area participating in the transfer. Both analyses provide examples for multiply optimized designs (symmorphosis): the bronchial tree generates an optimal oxygen reservoir at minimum energy dissipation; the acinar tree generates a near-optimal oxygen receptor at nearminimum energy dissipation. The dissipation results are a consequence of the uniform pressure drop across all bronchial ducts, and uniform concentration drop across almost all alveolar membranes (equipartition of thermodynamic forces), created by the spacefilling bronchial and acinar tree, respectively [26].

An equivalent formulation of efficient diffusion is: the lung operates near the transition from partial screening to no screening of the diffusion field, at which the diffusivity, permeability, and acinus structure match so that each oxygen molecule visits each membrane site essentially once, and only once, before crossing the membrane. We obtain this result by computing the oxygen current across the lung analytically, from a set of fractal power laws, based on minimal morphological and physicochemical input. We have validated the results by comparing them with numerically computed currents for two models of the acinar airways, and with experiment.

For respiration at rest, we find that the oxygen explores surface regions large compared to the subacinus entrance, but not the entire surface (partial screening II; oxygen enters deep into the alveolar system), while at exercise the oxygen visits the entire surface, possibly multiple times (no screening; oxygen enters the alveolar system completely). Thus, at rest and exercise, the lung operates on opposite sides of the screening transition. As the oxygen reservoir moves deeper into the acinar tree and feeds smaller diffusion spaces (1/8 and 1/128 acini, respectively) as we go from rest to 
exercise, but the current is to remain as close to the screening transition as possiblethe hallmark of an optimal gas-exchange design, the two diffusion spaces must operate on opposite sides of the screening transition. The switch from partial to no screening has previously been interpreted as a natural progression toward maximum respiratory efficiency, $\eta \sim 100 \%$ [21]; here we establish that the switch is necessary and sufficient for optimal gas exchange - an exchange in which all membrane sites participate and no oxygen molecule has to wait for transfer across the membrane.

We thank M. Filoche, B. Sapoval, and E.R. Weibel for valuable discussions. Support from the O.M. Stewart Fund $(\mathrm{CH})$, the Dutch National Science Foundation (SG and MOC), the National Institute of Health (VHH; R37 HL42528), the National Aeronautics and Space Administration (VHH; NAG 5-12300), the Visiting Professorship Program of Delft University of Technology (PP), and the Petroleum Research Fund, administered by the American Chemical Society (PP), is gratefully acknowledged.

\section{References}

[1] Mandelbrot B.B. The Fractal Geometry of Nature. San Francisco: Freeman, 1982.

[2] Bunde A., Havlin S. Fractals and Disordered Systems, $2^{\text {nd }}$ ed. New York: Springer, 1996.

[3] Sapoval B. Universalités et Fractales. Paris: Flammarion, 1997.

[4] Meakin P. Fractals, Scaling, and Growth Far from Equilibrium. Cambridge, UK: Cambridge University Press, 1998.

[5] In some cases a property may depend on additional structural parameters, such as lacunarity, spectral dimension, or the spectrum of roughness exponents [6].

[6] Gheorghiu S., Pfeifer P. Nonstandard roughness of terraced surfaces. Phys Rev Lett 2000; 85: 3894-3897.

[7] Pfeifer P., Gheorghiu S. Counterexamples in fractal roughness analysis and their physical properties. Int J Mod Phys B 2001; 15: 3197-3206.

[8] Pfeifer P. Characterization of surface irregularity. In: Laszlo P., ed. Preparative Chemistry Using Supported Reagents. San Diego: Academic Press, 1987; 13-33.

[9] Coppens M.-O., Froment G.F. Catalyst design accounting for the fractal surface morphology. Chem Engng J 1996; 64: 69-76.

[10] Gheorghiu S., Coppens M.-O. Optimal bimodal pore networks for heterogeneous catalysis. AIChE J 2004; 50: 812-820.

[11] Fawcett D.W. A Textbook of Histology, $12^{\text {th }}$ ed. New York: Chapman \& Hall, 1994.

[12] Weibel E.R. The Pathway for Oxygen. Cambridge, MA: Harvard University Press, 1984.

[13] Weibel E.R. Symmorphosis. Cambridge, MA: Harvard University Press, 2000.

[14] Martin P.M., Monzyk B.F., Burckle E.C., Busch J.R., Gilbert R.J., Dasse K.A. Thin films are helping fight against pulmonary diseases: development of a photolytic artificial lung. Vac Techn \& Coating 2004; Aug. 2004: 40-49.

[15] Weibel E.R. Fractal geometry: a design principle for living organisms. Am J Physiol 1991; 261: L361-L369.

[16] West B.J. Fractal Physiology and Chaos in Medicine. Singapore: World Scientific, 1990. 
[17] Shlesinger M.F, West B.J. Complex fractal dimension of the bronchial tree. Phys Rev Lett 1991; 67: 2106-2108.

[18] West G.B., Brown J.H., Enquist B.J. A general model for the origin of allometric scaling laws in biology. Science 1997; 276: 122-126.

[19] Mauroy B., Filoche M., Weibel E.R., Sapoval B. An optimal bronchial tree may be dangerous. Nature 2004; 427: 633-636.

[20] Sapoval B. Transfer to and across irregular membranes modeled by fractal geometry. In: Nonnenmacher T.F., Losa G.A, Weibel E.R., eds. Fractals in Biology and Medicine. Basel: Birkhäuser, 1994; 241-250.

[21] Sapoval B., Filoche M., Weibel E.R. Smaller is better-but not too small: a physical scale for the design of the mammalian pulmonary acinus. Proc Natl Acad Sci USA 2002; 99: 10411-10416.

[22] Felici M., Filoche M., Sapoval B. Diffusional screening in the human pulmonary acinus. J Appl Physiol 2003; 94: 2010-2016.

[23] Felici M., Filoche M., Sapoval B. Renormalized random walk study of oxygen absorption in the human lung. Phys Rev Lett 2004; 92: 068101-(1-4).

[24] Frauenfelder H. Complexity in proteins. Nature Struct Biol 1995; 2: 821-823.

[25] Murray C.D. The physiological principle of minimum work. I. The vascular system and the cost of blood volume. Proc Natl Acad Sci USA 1926; 12: 207214.

[26] Gheorghiu S., Kjelstrup S., Pfeifer P., Coppens M.-O. Is the lung an optimal gas exchanger? In this volume.

[27] Adam G., Delbrück M. Reduction of dimensionality in biological diffusion processes. In: Rich A., Davidson N., eds. Structural Chemistry and Molecular Biology. San Francisco: Freeman, 1968; 198-215.

[28] Kac M. Probabilistic methods in some problems of scattering theory. Rocky Mountain J Math 1974; 4: 511-537.

[29] Simon B. Functional Integration and Quantum Physics. New York: Academic Press, 1979; 231-245.

[30] Berg H.C., Purcell E.M. Physics of chemoreception. Biophys J 1977; 20: 193219.

[31] Makarov N.G. On the distortion of boundary sets under conformal mappings. Proc London Math Soc 1985; 51: 369-384.

[32] Pfeifer P., Sapoval B. Optimization of diffusive transport to irregular surfaces with low sticking probability. Mat Res Soc Symp Proc. 1995; 366: 271-276.

[33] Pfeifer P., Hagerty P.J. Screening transition in diffusion to and across fractal surfaces. In: Giona M., Biardi G., eds. Fractals and Chaos in Chemical Engineering. Singapore: World Scientific, 1997; 151-164.

[34] Filoche M., Sapoval B. A simple method to compute the response of nonhomogeneous and irregular interfaces: electrodes and membranes. J Phys I France 1997; 7: 1487-1498.

[35] Sapoval B., Filoche M., Karamanos K., Brizzi R. Can one hear the shape of an electrode? I. Numerical study of the active zone in Laplacian transfer. Eur Phys J B 1999; 9: 739-753.

[36] Sagan H. Space-Filling Curves. New York: Springer, 1994; 49-68.

[37] Haefeli-Bleuer B., Weibel E.R. Morphometry of the human pulmonary acinus. Anat Rec 1988; 220; 401-414. 